\title{
TÉCNICAS DE REABILITAÇÃO NA INATENÇÃO HEMIIESPACIAL SELECTIVA
}

\section{Clara de Santos Loureiro}

Resumo O neglect constitui o defeito cognitivo que, mais frequentemente, decorre de uma lesão do hemisfério cerebral direito. As suas manifestações clínicas são muito heterogéneas e acarretam ao doente graves repercussões na sua qualidade de vida. O facto de a sintomatologia mais evidente recuperar espontaneamente nos primeiros dez dias após a instalação da lesão, leva, possivelmente, à ideia generalizada, entre técnicos de saúde, de que o neglect tem geralmente um prognóstico favorável. Na realidade, as suas manifestações podem persistir, embora de forma subtil, durante muitos meses. Daí a importância da implementação de programas de reabilitação. As primeiras técnicas de reabilitação do neglect surgiram intuitivamente da prática clínica. Só mais tarde foi desenvolvido, de forma mais sistematizada, o treino da capacidade de pesquisa visuoespacial. Embora este tipo de técnica possa ter limitações quanto à generalização que proporciona para actividades funcionais, mostrou-se útil no treino de tarefas específicas. Um conjunto de outras técnicas tem sido implementado com o objectivo de minorar as manifestações do neglect, embora os resultados não sejam conclusivos. ${ }^{1}$

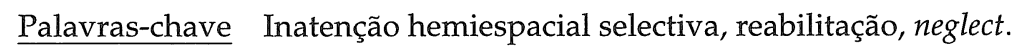

\section{Introdução}

\section{Definição}

A capacidade de explorar o espaço e, consequentemente, de orientar voluntariamente a atenção para estímulos relevantes, é um elemento fundamental no comportamento adaptativo do ser humano. A inatenção hemiespacial selectiva, ou neglect (termo utilizado na literatura anglo-saxónica, que será adoptado ao longo deste texto), decorre de uma lesão cerebral e consiste na perturbação dessa capacidade atencional, desde que a mesma não possa ser atribuída a um defeito sensorial ou motor. Repercute-se no indivíduo numa impossibilidade em atender, orientar-se para, ou responder, a estímulos novos ou signficativos (visuais, tácteis ou auditivos) que se lhe apresentem no hemiespaço contralateral à lesão (Heilman et al., 1993).

Clara de Santos Loureiro, Laboratório de Estudos de Linguagem, Centro de Estudos Egas Moniz, Hospital de Santa Maria. 


\section{Etiologia e neuropatologia}

O neglect surge, em grande parte dos casos, na sequência de patologias de instalação aguda, constituindo o acidente vascular cerebral (AVC) a causa mais comum para a sua ocorrência, ainda que tumores de rápido crescimento e traumatismos cranianos possam igualmente estar na sua origem (Heilman et al., 1993). Decorre, com mais frequência, de uma lesão do hemisfério direito, repercutindo-se, por conseguinte, as suas manifestaçōes no lado esquerdo do espaço. Calcula-se, pois, que esta patologia, que afecta cerca de $23 \%$ dos doentes vítimas de AVC unilateral em fase aguda, se manifesta em $42 \%$ dos doentes cuja lesão se situa no hemisferio cerebral direito e apenas em $8 \%$ dos afectados no hemisfério esquerdo (Pederson et al., 1997).

Considera-se actualmente que, subjacente ao mecanismo responsável pela maior potencialidade do hemisfério direito no controle da atenção espacial, haja um sistema paralelo de activação entre diferentes estruturas anatómicas desse hemisfério. Três áreas corticais têm especial importância na exploração do espaço: a região parietal posterior, os campos visuais frontais (frontal eyes fields, área 8 de Broadman) e a parte anterior do cíngulo (ou circunvolução do corpo caloso); estas áreas, além de estabelecerem ligaçōes entre si, relacionam-se também com estruturas subcorticais, como sejam os núcleos pulvinares do tálamo e o estriado (Mesulam, 1981, 1990). A integridade destas áreas e das suas conexões é, pois, fundamental para assegurar a capacidade de controlo da atenção espacial.

\section{Manifestaçōes clínicas}

As manifestaçōes clínicas do neglect são muito heterogéneas, não se podendo, por conseguinte, falar de uma síndroma unitária (Heilman et al., 1993; Stone et al., 1998). De facto, podem-se distinguir diferentes componentes no neglect: a) visuoespacial; b) motor; c) representacional. $O$ doente apresenta, então, respectivamente: a) uma incapacidade em pesquisar visualmente o espaço circundante; b) em desencadear os movimentos motores necessários à sua exploração; e c) em representá-lo mentalmente na sua totalidade.

Estes aspectos podem manifestar-se a um nível: 1) extrapessoal, quando os estímulos presentes no espaço circundante contralateral à lesão não são tidos em consideração pelo doente; 2) pessoal, se o doente deixa parte do corpo "esquecida" ao efectuar tarefas que geralmente exigem a sua utilização ou ao executar actividades que sobre elas recaem; e 3) representacional. Determinadas actividades podem, assim, tornar-se de difícil execução, já que o doente negligencia os estímulos que se encontram no seu hemiespaço esquerdo. Pode, por conseguinte, 1) nạoo evitar obstáculos quando se desloca, omitir palavras durante a leitura de um texto ou ignorar determinados alimentos enquanto toma uma refeiçăo; 2) ser-lhe impossível realizar actividades que recaiam sobre o seu corpo, 
como seja o fazer a barba ou o lavar a cara; 3) ter dificuldade na evocação de imagens mentais.

Encontram-se igualmente associados ao neglect o fenómeno de extinção, no caso de o doente, perante estímulos simultâneos, igualmente relevantes, negligenciar permanentemente o estímulo que se lhe apresente pela esquerda, e o de anosognosia, se o mesmo năo reconhecer os seus defeitos sensoriais primários.

\section{Historia natural}

As manifestações mais visíveis do neglect recuperam espontaneamente durante os primeiros 10 dias após a instalação da lesão (Stone et al., 1992). Assim sendo, assiste-se, nestes poucos dias, à transformação de uma síndroma com manifestações muito evidentes num quadro clínico discreto, cuja sintomatologia só poderá ser posta em evidência através de cuidadosa observação. Este facto está, possivelmente, na base da ideia generalizada, entre os técnicos de saúde, de que esta patologia tem geralmente um prognóstico favorável. No entanto, são muitos os casos descritos de doentes com neglect cujas manifestaçōes persistem mesmo durante vários meses, após a instalação da lesão (Sunderland et al., 1987).

\section{Repercussōes}

O neglect tem repercussões negativas não só na recuperação do doente, mas igualmente na sua qualidade de vida. De facto, a esta síndroma estão associados internamentos mais longos; estadias mais prolongadas nos centros de reabilitação; recuperações mais lentas, e nem sempre totais, dos defeitos motores e sensoriais; uma maior necessidade de assistência nas actividades da vida diária (Paolucci et al., 1996). Muitas vezes associada ao neglect, a existência de anosognosia constitui um factor de mau prognóstico na recuperaçâo do doente (Gianella \& Mattioli, 1992).

A persistência das manifestações do neglect e as sérias repercussões que estas acarretam ao doente, na execução das actividades de vida diária, apontam para a importância de programas de reabilitação que lhe possibilitem uma recuperação da sintomatologia e, por conseguinte, uma melhor qualidade de vida. 


\section{Reabilitação cognitiva do neglect}

Os primeiros passos da reabilitação cognitiva do neglect

As primeiras técnicas de reabilitação do neglect surgiram intuitivamente da prática clínica. As estratégias utilizadas pelos técnicos de saúde tinham como objectivo a recuperação dos sintomas, pela resolução do problema subjacente: a incapacidade do doente em orientar-se automaticamente para o hemiespaço esquerdo.

A estrutura dos programas de reabilitação e as técnicas de estimulação utilizadas

O tipo de intervenção que surge na sequência das primeiras abordagens adoptadas na reabilitação do neglect e que ainda hoje é utilizado, tem como principal objectivo a re-orientação da atenção do doente para o hemiespaço esquerdo. Para alcançar esse objectivo, dever-se-á, primeiramente, identificar as capacidades perturbadas que estão na base da condição patológica e, posteriormente, facultar ao doente um programa de reabilitação adequado. Espera-se, assim, que o treino numa determinada tarefa perceptiva acarrete uma generalização automática para outras actividades perceptivas semelhantes, e surta, igualmente, efeito nas actividades da vida diária (Calvanio et al., 1993).

Com o intuito de minorar o defeito atencional do doente, este tipo de intervenção recorre, frequentemente, a diferentes técnicas de estimulação, nomeadamente à estimulação visual lateralizada e ao feedback. A intervenção é estruturada de forma a que, numa fase inicial do tratamento, este tipo de estimulação seja constantemente utilizado e esteja presente em diferentes modalidades sensoriais; posteriormente, à medida que o paciente desenvolve, de forma autónoma, estratégias compensatórias, tal estimulação vai sendo reduzida.

\section{Estimulação visual lateralizada}

Muitas tarefas utilizadas nos programas de reabilitação do neglect recorrem ao uso de estimulação visual lateralizada (pistas visuais), com o intuito de proporcionar ao paciente pistas que lhe facilitem a orientação da atenção para o lado do espaço que ele negligencia. Quando utilizadas, estas pistas são geralmente estáticas símbolos coloridos, números ou letras - e estão dispostas no lado esquerdo dos estímulos que constituem a tarefa.

A presença deste tipo de estimulação parece ser eficiente na redução do neglect. Riddoch e Humphreys (1983) pediram a cinco doentes com neglect, decorrente de uma lesão do hemisfério direito, que executassem uma tarefa de bissecção de linhas, na presença e na ausência de uma pista estática, disposta no hemiespaço esquerdo. Verificaram uma marcada diminuição das manifestações da 
síndroma, quando os doentes eram forçados a identificar a pista estática antes de realizarem a tarefa.

No entanto, verificaram que este tipo de estimulação só surtia o efeito desejado se o doente fosse instruído a procurar essa pista; se tal não acontecesse, o comportamento de negligência mantinha-se (Riddoch \& Humphreys, 1983).

Uma vez que o doente com neglect não tem capacidade para orientar a atenção, de forma automática, para o hemiespaço esquerdo, alguns autores consideram que esta técnica não é particularmente eficiente, uma vez que exige ao doente que tome a iniciativa de adoptar uma estratégia para compensar o seu defeito, do qual pode nem ter consciência. Sugerem, por conseguinte, que o uso de pistas dinâmicas poderá ser mais benéfico como forma de estimulação.

Para avaliar a maior eficácia das pistas dinâmicas em relação às estáticas Butter et al. (1990) compararam o desempenho de 18 pacientes com neglect, decorrente de uma lesão no hemisfério direito, em duas tarefas de bissecção de linhas, que apenas diferiam entre si na existência, à esquerda de cada uma das linhas, de estímulos visuais dinâmicos ou estáticos. Verificaram que, embora o estímulo estático tenha produzido um efeito positivo na redução das manifestações de neglect, o estímulo dinâmico produziu uma melhoria mais significativa.

Num estudo posterior, Butter e Kisch (1995) corroboraram estes resultados, ao constatarem uma diminuição significativa do comportamento de neglect em 11 doentes, graças à presença de pistas dinâmicas. Utilizaram uma tarefa computorizada de pesquisa visual, em que o doente deveria encontrar uma letra-alvo no meio de letras distractoras, enquanto estímulos visuais dinâmicos eram continuamente apresentados no lado esquerdo do ecrã.

Os resultados obtidos, em ambos os estudos, permitiram concluir que a estimulação visual dinâmica é particularmente eficaz na redução das manifestações do neglect.

\section{Feedback}

Para além do uso da estimulação visual lateralizada, o feedback é também considerado útil no treino de pacientes com neglect.

A forma mais usualmente utilizada de feedback é a auditiva. O terapeuta, durante as sessões de reabilitação, orienta constantemente, de forma explícita, a atenção do doente para o lado do espaço por si negligencido; ou seja, leva-o, através de ordens verbais, a olhar para a esquerda, sempre que o mesmo não pesquisa esse hemiespaço enquanto executa determinadas tarefas.

Os estímulos sonoros constituem uma outra forma de feedback auditivo utilizado em terapia; são considerados igualmente úteis para atrair a atenção do doente para o hemiespaço negligenciado.

Ao contrário dos doentes com defeito de campo, que desenvolvem estratégias de compensação, de forma a pesquisarem o espaço circundante na sua 
totalidade, os doentes com neglect não orientam os olhos para o campo visual contralateral à lesão (Ishiai et al., 1987). Fanthom et al. (1995), partindo desta evidência, procuraram investigar a possibilidade da redução das manifestações do neglect, no caso dos doentes serem informados àcerca da orientação dos seus olhos. Para tal, compararam dois grupos de doentes com neglect decorrente de uma lesão no hemisfério direito. Aos doentes de um dos grupos foi fornecido, durante as sessões de terapia, um sinal sonoro (feedback auditivo) sempre que o seu olhar não era direccionado para a esquerda durante um intervalo de 15 segundos (informação avaliada através de uns óculos detectores de movimento, responsáveis, igualmente, pela emissão do sinal sonoro); aos do outro não foi dado qualquer tipo de feedback. Ao não verificarem qualquer diferença entre os dois grupos, os autores concluíram que esta técnica não tivera qualquer efeito, tanto na orientação dos olhos, quanto na execução dos testes do behavioural innatention test (BIT) (uma bateria de testes amplamente utilizada na avaliação do neglect).

Um outro tipo de feedback utilizado nos programas de reabilitação é o que procura confrontar o doente com o seu próprio defeito. Alguns autores consideram que consciencializar o doente do seu defeito de exploração do espaço é fulcral para obter dele a colaboração necessária ao sucesso do programa de reabilitação e para o dotar de um papel activo no treino das estratégias compensatórias. Sugerem, pois, que só eliminando o fenómeno de anosognosia, muitas vezes associado à síndroma do neglect, se torna possível a implementação de um treino eficiente, em que as estratégias adoptadas surtem o efeito desejado (McGlynn \& Schacter, 1989).

Com esse objectivo, muitos terapeutas confrontam o doente com o resultado do seu desempenho nas tarefas por si realizadas, guiando, de forma verbal, a sua atenção para o hemiespaço negligenciado, de forma a que o mesmo se aperceba que, enquanto executava a tarefa pedida, ignorou uma parte do espaço; recorrem ainda a técnicas com suporte visual, como, por exemplo, o espelho.

Alguns autores têm investigado a utilidade de outro tipo de feedback com suporte visual: a gravação em vídeo do desempenho do doente durante a realização de determinadas tarefas. Consideram que esta técnica pode fornecer ao doente uma percepção mais precisa do seu defeito, uma vez que a gravação em vídeo, ao contrário do espelho, proporciona ao paciente a possibilidade de observar o seu comportamento - de negligência do hemiespaço esquerdo - no lado direito do monitor de TV, e, consequentemente, de aperceber-se dos seus erros (fornece-lhe uma imagem invertida de si próprio). Permite, portanto, a obtenção de imagens objectivas e concretas, que possibilitam uma análise imediata do seu conteúdo (Soderback et al., 1992; Tham \& Tegnér, 1997).

Soderback et al. (1992), com o objectivo de avaliar a eficácia deste tipo de feedback, compararam o desempenho de quatro doentes com neglect decorrente de um AVC do hemisfério direito. Pediram aos doentes que realizassem três tarefas domésticas e um teste de barragem de linhas; foi ainda preenchida uma escala funcional. O desempenho dos doentes durante a realização das tarefas domésticas foi filmado e posteriormente visionado por cada paciente, sob a orientação de um terapeuta. O conteúdo do filme obtido foi analisado, de forma a que cada doente se 
apercebesse de que tinha ignorado uma parte do espaço enquanto realizava a tarefa, e foram discutidas as estratégias que cada um poderia adoptar para lidar com a situação. Os autores verificaram que, ao longo de quatro sessões de treino, todos os pacientes melhoraram na tarefa treinada; não registaram, no entanto, qualquer alteração no desempenho, quer do teste de barragem de linhas, quer na escala funcional.

Igualmente interessados nas potencialidades desta técnica, Tham e Tegnér (1997) compararam o grau de eficácia na redução das manifestações de neglect do feedback através de imagens obtidas em vídeo e do feedback convencional (em que o doente era confrontado com o seu comportamento de negligência através de pistas verbais e visuais) durante uma tarefa doméstica. Avaliaram, ainda, se essa melhoria se generalizava a outros testes de neglect. Para tal, recorreram a um grupo de 14 pacientes com esta patologia, decorrente de um AVC no hemisfério direito; sete pacientes foram treinados através do feedback das imagens obtidas em vídeo e os outros sete através do feedback convencional. Verificaram que o primeiro grupo obteve resultados significativamente melhores na realização da tarefa, embora não tenham constatado, em nenhum dos dois grupos, uma generalização para os outros testes de neglect. Os autores consideraram que a inexistência de generalização pode ser devida ao facto de uma simples sessão de treino não ser suficiente para permitir a transferência das estratégias de compensação adquiridas para outras tarefas.

Estes estudos demonstraram que, apesar de não se ter verificado uma generalização das estratégias adquiridas, a tomada de consciência por parte do doente do defeito de exploração espacial é de extrema importância na redução das manifestações do neglect.

\section{Treino da capacidade de pesquisa visuoespacial}

Uma das primeiras intervenções levadas a cabo na tentativa de reabilitar o neglect foi descrita por Lawson (1962). Com o intuito de treinar a capacidade de leitura de dois doentes com neglect, o autor relembrava-os constantemente que deviam olhar para a esquerda sempre que o conteúdo semântico da frase não fizesse sentido, e que deviam seguir o texto com o indicador. Verificou, como resultado deste treino, que os dois doentes apresentavam uma franca melhoria na capacidade de leitura. Não constatou, no entanto, uma generalização para outras capacidades; de facto, não registou quaisquer alterações nas outras capacidades visuoespaciais avaliadas (cópia de desenhos). O autor concluiu que o benefício deste tipo de treino se cingia apenas às tarefas treinadas.

O estudo de Lawson constitui uma descrição pontual deste tipo de intervenção. De facto, é nos anos 70 e 80 que um grupo de investigadores de Nova Iorque desenvolve, de forma mais sistematizada, o treino da capacidade de pesquisa visuoespacial (Weinberg et al., 1977). 
Este tipo de intervenção tinha como principal objectivo minorar a sintomatologia clínica resultante da lesão cerebral; o alvo dos programas de reabilitação eram, portanto, as manifestações do neglect, e não a compreensão dos mecanismos neuropatológicos subjacentes. Visava, por conseguinte, através de técnicas específicas, dotar o doente da capacidade de orientar a atenção para o hemiespaço por si negligenciado. Para tal, baseava-se nos princípios da psicologia comportamental e na teoria da aprendizagem, que postulam que a apresentação simultânea de um determinado estímulo e de um tipo de resposta específico permite criar no doente um condicionamento, de tal forma que a resposta se torna automática (é interiorizada pelo doente e funciona como uma "ordem interna"), perante uma tarefa semelhante à treinada, mesmo que o estímulo deixe de estar presente.

O grupo de investigadores desenvolveu, então, um conjunto de técnicas, que se vieram a tornar muito influentes nos anos subsequentes, estruturadas de forma a induzir, de forma gradual, o doente a orientar a atenção para o hemiespaço esquerdo.

O board scanning é um exemplo de uma das técnicas por eles criada; consiste num tabuleiro rectangular, disposto na horizontal, com lâmpadas em toda a sua extensão. Foi construído desta forma para proporcionar ao doente uma tarefa de pesquisa visuoespacial; de facto, cabia ao doente a tarefa de seguir, com os olhos, a sequência de luzes manipuladas pelo investigador. Na fase inicial do treino, o doente era induzido a acompanhar uma sequência que ocorria apenas no hemiespaço direito do doente (a primeira luz a ser acesa era a situada na parte central do tabuleiro; depois, as luzes eram acesas, uma a uma, em direcção à extremidade direita); numa fase mais avançada, o doente era levado a seguir, de forma progressiva, sequências de luzes que abrangiam o hemiespaço esquerdo.

No treino de outro tipo de tarefas, como, por exemplo, a leitura, o grupo de investigadores recorreu amplamente à estimulação visual lateralizada (pistas estáticas: símbolos coloridos ou números no início de cada linha do texto apresentado) e ao feedback auditivo ("olhe para a esquerda para encontrar o início de cada linha"), como forma de facilitar o treino; a densidade do texto foi também manipulada (tamanho das frases e da letra em que estavam impressas). Weinberg et al. (1977) verificaram que num grupo de 57 pacientes com neglect, decorrente de um AVC no hemisfério direito, os 25 doentes que foram submetidos a um treino específico da capacidade de leitura revelaram resultados francamente melhores na tarefa treinada, do que o grupo de controlo, cujo treino foi inespecífico; constataram ainda uma generalização para outras actividades.

Depois destes trabalhos, muitos foram os investigadores a interessarem-se sobre a potencialidade do treino da capacidade de exploração visuoespacial como técnica de reabilitação do neglect. Tal como Lawson (1962) e o grupo de investigadores de Nova Iorque, muitos clínicos centraram os seus programas de reabilitação no treino de capacidades académicas, como a leitura e a escrita; outros focaram-se no treino de actividades funcionais. 
Embora os estudos realizados variem em diversos aspectos, e daí surja, provavelmente, a falta de uniformidade dos resultados obtidos, todos procuraram: a) direccionar de forma explícita a atençăo do doente para o lado esquerdo do espaço; b) fomentar mudanças lentas e progressivas; e c) reforçar de modo positivo o doente, como forma de estabilizar a aprendizagem. Além de procurarem testar a eficácia deste tipo de treino e das técnicas desenvolvidas pelo grupo de Nova Iorque, os autores procuraram investigar o problema da generalização, levantado, desde cedo, por Lawson.

Webster et al. (1984) recorreram às técnicas desenvolvidas pelo grupo de Nova Iorque e verificaram que, embora se tenha constatado uma melhoria no desempenho de doentes com neglect, decorrente de um AVC no hemisfério direito, na tarefa de pesquisa do board scanning, não se registaram alteraçōes em tarefas năo treinadas, como a leitura ou a barragem de letras; o oposto revelou-se igualmente verdade.

Também Robertson et al. (1990) se propuseram avaliar a eficácia do treino específico e estruturado da capacidade de pesquisa visuoespacial. Para tal, dividiram 36 pacientes com neglect unilateral, determinado pelos testes comportamentais do BIT, em dois grupos aleatórios, e compararam os resultados do primeiro grupo, com 20 doentes, submetido a um treino computorizado da atençăo e da capacidade de explorar o espaço, com os do segundo, com 16 individuos, sujeito a um programa de computador recreativo (jogos de palavras). A comparação dos dois grupos não revelou diferenças estatísticas significativas entre ambos.

Wagenaar et al. (1992) procuraram avaliar a eficácia do treino da capacidade de pesquisa visuoespacial numa tarefa de leitura e na capacidade de o doente se deslocar numa cadeira de rodas. Procederam a três fases de treino; depois de cada uma dessas fases o desempenho dos doentes foi avaliado (através de um teste de pesquisa visual computorizado, um teste de bisseç̧ão de linhas e um teste de barragem de letras). Quatro dos cinco pacientes treinados mostraram uma melhoria na capacidade de pesquisa visual na tarefa de leitura; não registaram um efeito positivo na deslocação em cadeira de rodas. Os autores concluíram que não havia evidência de uma generalização do treino para outros domínios funcionais.

Pizzamiglio et al. (1992), igualmente interessados nesta problemática, obtiveram resultados diferentes. De facto, verificaram que o treino da capacidade de pesquisa visuoespacial de 32 doentes com neglect crónico, decorrente de uma lesão do hemisfério direito, produziu uma melhoria significativa no seu desempenho, quando testados com instrumentos estandardizados para avaliação do neglect. As tarefas utilizadas no treino exigiam ao paciente que fizesse uma pesquisa sistematizada do espaço, de forma a produzir respostas correctas (encontrar um número em diferentes porções do espaço; ler ou copiar frases; desenhar e descrever figuras). No início do treino o doente era provido de um grande número de pistas, através de feedback auditivo e de estimulação visual lateralizada, e a própria complexidade dos estímulos era reduzida; ao longo das sessōes estas pistas foram sendo retiradas e os estímulos tornaram-se mais 
complexos. Além de verificarem uma melhoria das manifestações do neglect, os autores constataram ainda, através do preenchimento de uma escala funcional, uma generalização para situações semelhantes àquelas vividas no dia-a-dia (informação corroborada pelos familiares). Sete pacientes foram avaliados aproximadamente 5 meses após o término da intervenção e obtiveram resultados semelhantes aos conseguidos anteriormente.

Num estudo posterior, Antonucci et al. (1995) corroboraram estes resultados. Compararam um grupo de doentes, sujeito a um treino específico da capacidade exploratória, com outro, submetido a treino inespecífico (puzzles, xadrez, jogos de cartas, palavras cruzadas). O treino específico decorreu nos moldes descritos no estudo anterior, já referido. Verificaram, com base numa bateria de testes estandardizados e numa escala funcional, uma melhoria mais significativa do primeiro grupo em relação ao segundo, que posteriormente, submetido a um treino específico, obteve os mesmos resultados encorajadores. Os autores concluíram, pois, que os resultados foram benéficos e significativos, e que se generalizaram a situações semelhantes àquelas que fazem parte da vida diária do doente.

Estes autores consideram que a ausência de generalização verificada em grande parte dos estudos pode estar relacionada com o tempo investido no treino das tarefas em questão (1-2 semanas). De facto, os estudos em que se constatou, não só uma melhoria das tarefas treinadas, mas igualmente uma generalização para outras actividades recorreram a um período de treino mais longo (5-8 semanas).

Levantam, pois, o problema da duração do tratamento. Da sua experiência concluem que, quando o programa de reabilitação tem início, muitos dos pacientes com neglect não estão conscientes do seu defeito e as mudanças conseguidas são transitórias e podem apenas ser obtidas com grande quantidade de estimulação. À medida que os pacientes tomam consciência do defeito, começam a desenvolver estratégias compensatórias e, gradualmente, vão deixando de necessitar do auxílio fornecido pelas pistas; os exercícios contínuos, com menor grau de estimulação, permitem-lhes tornar automática a capacidade de pesquisa visuoespacial. Para que estas fases possam ocorrer, os autores consideram necessário que a estrutura dos procedimentos postos em prática seja adequada e que haja um tempo mínimo de treino. Referem ter verificado que interrupções durante o processo de reabilitação (devido a problemas médicos ou outros) atrasaram o processo de aprendizagem.

Embora não esteja ainda esclarecido se se verifica, de facto, uma generalização para situações funcionais a partir do treino da capacidade de pesquisa visuoespacial, há alguns benefícios terapêuticos deste tipo de reabilitação que não apresentam dúvidas. De facto, revela-se de extrema utilidade na produção de respostas específicas (sacadas compensatórias) em situações treinadas (como, por exemplo, a leitura e a escrita). Aspectos específicos do comportamento do doente, importantes para a sua recuperação funcional, podem, por conseguinte, tornar-se alvos deste tipo de intervenção, conferindo-lhe uma maior autonomia e, consequentemente, uma maior qualidade de vida. 


\section{Outras técnicas utilizadas na reabilitação do neglect}

Ao longo dos anos uma panóplia de outras técnicas têm surgido como forma de reabilitar o neglect. Os mecanismos subjacentes a esta síndroma não são ainda conhecidos, mas existem já uma série de possíveis teorias explicativas. É precisamente com base em algumas dessas teorias que tais técnicas se têm proposto minorar as manifestações desta síndroma. Serão descritas, de seguida, algumas dessas técnicas, embora muitas outras estejam referidas na literatura.

\section{Treino da capacidade de atenção sustentada}

A atenção sustentada refere-se à capacidade do indivíduo em se manter num estado de alerta num ambiente em que os estímulos não apresentam qualquer novidade. Um defeito nesta capacidade pode manifestar-se pela dificuldade de concentração, que alguns doentes revelam, durante a execução de tarefas que apresentam uma certa monotonia.

O hemisfério direito, em particular as áreas frontoparietais, é responsável pela integridade desta capacidade. Este dado foi demonstrado em estudos de activação cerebral, durante a execução de tarefas que exigiam ao indivíduo que mantivesse a atenção sustentada (Pardo et al., 1991).

Posner (1993) considera que a capacidade de orientar a atenção para um determinado sector do espaço depende de um sistema de orientação espacial, parcialmente localizado no lobo parietal direito. A eficácia desse sistema é modulado pelo sistema de atenção sustentada. Se assim for, o treino desta capacidade possibilitará a redução das manifestações do neglect.

Robertson et al. (1995) treinaram um grupo de pacientes com neglect, que apresentavam também um defeito na capacidade de atenção sustentada, a controlarem voluntariamente, de forma verbal, a atenção investida durante a execução de diferentes tarefas. Os pacientes foram instruídos a adoptaram, de forma sistemática, um "sistema de aviso" verbal que lhes permitisse aumentar a concentração na tarefa a realizar, até que o mesmo se tornasse automático. Os resultados obtidos foram muito encorajadores; não só verificaram uma melhoria na capacidade de atenção sustentada, mas também uma redução das manifestações do neglect.

Os autores referem ter verificado que os doentes com uma perturbação na capacidade de atenção sustentada têm consciência do seu defeito, o que nem sempre acontece com as manifestações de neglect, pelo que, mais facilmente, compreendem a importância de implementar estratégias que melhorem o seu desempenho. 


\section{Treino com recurso à utilização da mão esquerda}

Além dos defeitos cognitivos que podem surgir na sequência de um AVC, os defeitos neurológicos, como a hemíparésia e a hemianópsia, são muito frequentes. De facto, muitos doentes com neglect apresentam uma parésia motora, que lhes impossibilita o uso da mão esquerda. Devido a este facto, utilizam exclusivamente a mão direita para realizar as tarefas perceptivo-motoras que fazem parte da avaliação do neglect (testes de papel e lápis).

- Alguns autores sugerem que as manifestações de neglect são o resultado do envolvimento selectivo do hemisfério esquerdo, que controla o movimento da mão direita, cuja activação deixou de ser inibida, pela lesão ocorrida no hemisfério direito (Kinsbourne, 1993).

Halligan \& Marshall (1989) descreveram o caso de um doente que, após uma lesão no hémisfério direito, adquiriu a síndroma do neglect, sem revelar qualquer defeito neurológico associado. Pôde, portanto, quando lhe foi pedido, utilizar a mão esquerda na execução das provas para avaliar o neglect. Os autores verificaram que o doente evidenciava um neglect marcado, num teste de bissecção de linhas e de barragem de estrelas, quando realizava as tarefas com a mão direita, e que o seu desempenho melhorou significativamente quando foi induzido a utilizar a mão esquerda.

Perante estás evidências, alguns autores põem a hipótese de que a redução das manifestações de neglect, pela utilização da mão esquerda, durante a realização de diversas tarefas, pode ser explicada pelo facto de o movimento da mão esquerda, constituir, por si só, uma fonte de estimulação visual lateralizada, que facilitaria ao doente a orientação da atenção para esse lado. Para testar esta hipótese Halligan et al. (1990) pediram a um paciente que executasse uma tarefa de bissecção de linhas, primeiro com a mão direita e, de seguida, com a esquerda. Verificaram que o uso da mão esquerda originava, de facto, a redução do neglect; no entanto, constataram ainda que quando o doente foi instruído a colocar a mão esquerda no lado direito de cada uma das linhas a bissectar, antes de executar a tarefa, o comportamento de negligência mantinha-se. Os autores concluíram que, quando a atenção do doente era primeiramente atraída para o lado direito, a vantagem de usar a mão esquerda desaparecia.

Um outro estudo foi levado a cabo por Robertson e North (1992). Os investigadores compararam o desempenho de um doente, enquanto executava uma tarefa de barragem de letras, em três situações diferentes: numa aplicação normal do teste; enquanto fazia movimentos com os dedos da mão esquerda, estando esta colocada na extermidade esquerda da folha de papel; enquanto era instruído a procurar o braço esquerdo antes de executar a tarefa, braço esse igualmente posicionado na extermidade esquerda da folha de papel. Verificaram que apenas se verificou uma redução do neglect quando o doente, ao mesmo tempo que executava a tarefa, mexia os dedos da mão esquerda. Estes resultados permitiram aos autores do estudo concluir que o recurso a pistas visuais dinâmicas é mais eficaz na redução das manifestações desta patologia. 


\section{Rotação do tronco}

A linha média do tronco parece ser o principal eixo de referência que o indivíduo adopta, quando estabelece coordenadas internas egocêntricas; são essas coordenadas que lhe permitem determinar, de forma subjectiva, o modo como os objectos estão distribuídos no espaço. Na realidade, é a linha média do tronco que, funcionando como eixo de referência, permite ao indivíduo estabelecer os limites entre um sector do espaço à sua esquerda e um sector à sua direita. Como tal, parece ser um factor decisivo, para determinar a porção do espaço negligenciada em pacientes com neglect e a porção do espaço não negligenciada (Karnath et al.,1991; Beschin et al., 1997).

Karnath et al. (1991) demonstraram que uma simples rotação do corpo de 15 . $^{\circ}$ para a esquerda podia reduzir significativamente as manifestações de neglect. Verificaram que a rotação do corpo (estando a cabeça e olhos direccionados para a frente) modificou a distribuição dos estímulos no espaço; de facto, os estímulos visuais que, na ausência da rotação do tronco, se encontravam à esquerda da linha média definida pelo tronco, passaram a estar localizados à direita dessa mesma linha, quando o doente foi induzido a fazer uma rotação do tronco para a esquerda. Os limites do espaço sofreram, pois, uma alteração, e os estímulos, anteriormente negligenciados, passaram a ser alvo da atenção dos doentes.

Mais recentemente Wiart et al. (1997) descreveram os resultados obtidos da combinação desta técnica com os métodos de treino de pesquisa visuoespacial desenvolvidos pelo grupo de investigadores em Nova Iorque. A tarefa de pesquisa visuoespacial, atribuída a um grupo de doentes com neglect, decorrente de um AVC do hemisfério direito, foi criada de forma a que o doente tivesse que fazer a exploração do espaço com o tronco; para tal foi preso ao tronco de cada doente um ponteiro. Os resultados obtidos revelaram uma redução das manifestações de neglect, não só em doentes com neglect na fase aguda do AVC, mas também em doentes cujo neglect se tornou crónico, e uma generalização para actividades funcionais.

\section{Estimulação sensorial}

O sistema vestibular é responsável pela integração da informação recebida através de três canais sensoriais distintos — o vestibular, o visual e o proprioceptivo - , de forma a controlar e modular a postura corporal do indivíduo. O mapa espacial do indivíduo parece, pois, ser assistido por esses três subsistemas, que geram as coordenadas espaciais de que o indivíduo necessita para explorar o espaço pessoal e extrapessoal. Esses subsistemas são independentes, pelo que não necessitam de ser activados em simultâneo. No caso de uma lesão focal, a desconexão nos circuitos que suportam um ou mais de tais subsistemas pode originar uma alteração da percepção subjectiva que o doente tem da posição dos objectos em relação ao seu corpo (Karnath, 1996). 
A linha média do corpo, tal como já foi referido, parece ser o principal eixo de referência que condiciona a forma como as coordenadas internas egocêntricas são determinadas pelo individuo. No indivíduo com neglect, a percepção subjectiva que o doente tem do posicionamento dos objectos em relaçăo à posição do corpo está perturbada.

Alguns autores analisaram a hipótese de a estimulação dos sistemas neurofisiológicos envolvidos no processo de organizaçăo de cooredenadas espaciais produzir uma redução do defeito de exploraçăo espacial. Verificaram que a activaçẩo de qualquer dos subsistemas, acima mencionados, é suficiente para reduzir ou, pelo menos, compensar, de forma parcial, as manifestaçōes do neglect, durante a execuçăo de tarefas de pesquisa visuoespacial. De facto, constataram que os três tipos de reflexo que se seguem à estimulação calórica e optocinética e à vibração dos músculos do pescoço, parecem modificar a forma como o indivíduo codifica a informaçăo espacial $\mathrm{e}$ conduzem temporariamente à remissão das manifestaçōes do neglect.

\section{Estimulaçâo calórica}

Para se proceder à estimulação do sistema vestibular, alguns investigadores procederam à estimulaçăo calórica, uma técnica utilizada na prática clínica para avaliar a integridade do reflexo ocular vestibular. Consiste numa estimulaçăo do ouvido interno, obtida através do contacto com água gelada ou quente. A estimulaçăo através de água fria produz um reflexo vestibulo-ocular com uma fase lenta de nistagmo em direç̧ăo ao ouvido estimulado, e um reflexo vestibulo-espinal, que se reflecte na orientação da postura na mesma direç̧ão; a estimulaçăo através de água quente tem o efeito contrário (Rubens, 1985).

Diversos estudos procuraram investigar a potencialidade da estimulação calórica como técnica para tratar o neglect. Para minorar as manifestaçōes desta síndroma, o teste calórico consistiria na estimulação do ouvido esquerdo do paciente, através de água fria.

Demonstraram uma reduçăo significativa das manifestaçôes clínicas, e atribuíram-na ao desvio dos olhos e da postura na direç̧ão oposta ao desvio patologicamente adquirido, em consequência da lesão cerebral.

Rubens (1985) estudou 18 pacientes com neglect extrapessoal, vitimas de AVC no hemisfério direito, e observou o seu desempenho em tarefas de pesquisa visuoespacial (contagem das pessoas presentes na sala; leitura de palavras multissilábicas; teste de barragem de linhas) antes, durante e cinco minutos depois da estimulaçăo calórica. Verificou que, à excepçăo de um doente, todos os outros melhoraram o seu desempenho durante a realizaçalo das tarefas, tendo-se verificado uma orientaçăo do olhar e do braço direito para a esquerda. No entanto, estas melhorais revelaram-se transitórias e a maior parte dos sintomas regressou passados poucos minutos. Concluiu que, mesmo em casos graves de neglect, o 
reflexo vestibular induzido pela estimulação calórica produziu resultados positivos e significativos.

Posteriormente, Cappa et al. (1987) demonstraram o efeito positivo da estimulação calórica sobre outras manifestações do neglect, nomeadamente sobre o neglect pessoal e a anosognosia. Rode e Perenin (1994) verificaram que a estimlação calórica pode ter também um efeito positivo na redução do neglect representacional. Pediram a oito doentes com neglect representacional que enumerassem o maior número possível de cidades de França, imaginando que uma linha vertical a dividia. O número de cidades referido pelos pacientes foi muito menor à esquerda do que à direita da linha imaginária; durante a estimulação calórica, todos os pacientes melhoraram as suas pontuações, quando referiram os nomes das cidades posicionadas à esquerda da linha imaginária.

Estes estudos vieram demontrar que a redução do neglect, através desta técnica, não pode ser explicada exclusivamente pela orientação dos olhos e da cabeça para a esquerda.

\section{Estimulação optocinética}

Um outro método que produz um nistagmo, semelhante ao produzido pela estimulação calórica, é a estimulação optocinética. Este reflexo surge normalmente quando o doente olha para um padrão em movimeto lento. Pizzamiglio et al. (1990) compararam três grupos de sujeitos - um grupo de doentes, com lesão uma no hemisfério direito, sem manifestações de neglect; outro, igualmente com uma lesão no hemisfério direito, mas com manifestações de neglect; e um grupo de controlo. Pediram aos indivíduos que efectuassem uma tarefa de bissecção de linhas na presença de um padrão em movimento e de outro fixo. Na presença do estímulo em movimento verificaram um nistagmo que originou uma alteração da percepção subjectiva da orientação do corpo, reduzindo, no caso dos doentes com neglect, as suas manifestações.

Concluíram que, dada a simplicidade do procedimento e o reduzido grau de desconforto produzido no doente, em comparação com a estimulação calórica, este procedimento poderá constituir uma técnica útil no tratamento do neglect.

\section{Estimulação dos músculos do pescoço}

Vários investigadores têm avaliado a eficácia da estimulação dos músculos do pescoço, através de vibração, na redução das manifestações de neglect. As descrições efectuadas relatam uma remissão temporária do defeito de pesquisa visuoespacial, em praticamente todos os doentes submetidos à estimulação (Karnath el al., 1993; Vallar et al., 1995; Vallar et al., 1996). 
O processo através do qual os diferentes tipos de estimulação sensorial produzem a remissão temporária do neglect não é ainda claro e a sua utilidade como técnica de reabilitação desta patologia está ainda longe de estar estabelecida.

\section{Terapếutica farmacológica}

Subjacente à capacidade de atenção e exploração visuoespacial está uma rede neuronal em larga escala, composta por redes neuronais regionais que, embora distantes, se encontram interligadas. A literatura sugere que as vias dopaminérgicas desempenham um papel de extrema importância na modulação desta rede neuronal.

A existência de estudos que investiguem a potencialidade de uma intervenção farmacológica na reduçăo das manifestações de neglect é escassa. Fleet et al. (1987) mostraram uma melhoria do neglect em dois pacientes (um com neglect crónico e outro na fase aguda) tratados com um agonista da dopamina. Referem, no entanto, a necessidade de se efectuarem outros estudos com um maior número de pacientes.

\section{Conclusões}

A eficácia das técnicas desenvolvidas, ao longo dos tempos, com o intuito de reabilitar o neglect, não está ainda determinada. Um aspecto importante na avaliação de um programa de treino é a generalização da melhoria do paciente. Os resultados obtidos não são conclusivos, uma vez que na literatura existem muitos estudos, cujas descriçōes năo săo concordantes.

$\mathrm{Na}$ base da falta de uniformidade dos resultados obtidos nos estudos referidos estão, provavelmente, diferenças metodológicas, nomeadamente no que diz respeito à selecçăo dos pacientes a incluir nos programas de treino, aos testes escolhidos para avaliar a recuperação do neglect e ao tempo destinado à reabilitação.

Anecessidade de estudos que controlem estas variáveis continua premente.

Nota

1 Morada para correspondência: Dra. Clara de Santos Loureiro, Laboratório de Estudos de Linguagem, piso 8, Centro de Estudos Egas Moniz, Hospital de Santa Maria, 1600 Lisboa; telefone/fax: 217934480; e-mail: labling@mail.telepac.pt. 


\section{Referências}

Antonucci, G., Guariglia, C., Judica, A., Magnotti, L., Paolucci, S., Pizzamiglio, L., \& Zoccolotti, P. (1995). Effectiveness of neglect rehabilitation in a randomized group study. Journal of Clinical and Experimental Neuropsychology, 17 (3), 383-389.

Beschin, N., Cubelli, R., Dellasala, S. \& Spinazzola, L. (1997). Left of what: The role of egocentric coordinates in neglect. Journal of Neurology, Neurosurgery \& Psychiatry, 63 (4), 483-489.

Butter, C. M., \& Kisch, N. (1995). Effect of lateralized kinetic visual cues on visual search in patients with unilateral spatial neglect. Journal of Clinical and Experimental Neuropsychology, 17, 856-867.

Butter, C. M., Kisch, N., \& Reeves, G. (1990). The effect of lateralized dynamic stimuli on unilateral spatial neglect following right hemisphere lesions. Restorative Neurology and Neuroscience, 2, 39-46.

Calvanio, R., Levine, D. N., \& Petrone, D. (1993). Elements of cognitive rehabilitation after right hemisphere stroke. Behavioural Neurology, 11, 25-57.

Cappa, S., Strerzi, R., Vallar, G., \& Bisiach, E. (1987). Remission of hemineglect and anosognosia during vestibular stimulation. Neuropsychologia, 25 (5), 775-782.

Fanthome, Y., Lincoln, N., Drummond, A., \& Walker, M. (1995). The treatment of visual neglect using feedback of eye movements: A pilot study. Disability and Rehabilitation, 17, 413-417.

Fleet, W. S., Valenstein, E., Watson, R. T., \& Heilman, K. M. (1987). Dopamine agonist therapy for neglect in humans. Neurology, 37, 1765-1771.

Gianella, B., \& Mattioli, F. (1992). Anosognosia e extrapersonal neglect as predictors of functional recovery following right hemisphere stroke. Neuropsychological Rehabilitation, 2, 169-178.

Halligan, P. W., Manning, L., \& Marshall, J. C. (1990). Hemispheric activation vs. spatio-motor cueing in visual neglect: A case study. Neuropsychologia, 29, 165-176.

Halligan, P. W. \& Marshall, J. C. (1989). Laterality of motor response in visuospatial neglect: A case study. Neurophychologia, 27, 1301-1307.

Heilman, K. M., Watson, R. T., \& Valenstein, E. (1993). Neglect and related disorders. In K. M. Heilman \& E. Valenstein (Eds.), Clinical Neurophychology (pp. 279-336). Nova Iorque: Oxford University Press.

Ishiai, S., Furukawa, T., \& Tsukagoshi, H. (1987). Eye fixation patterns in homonymous hemianopia and unilateral spatial neglect. Neuropsychologia, 25, 675-679.

Karnath, H. O. (1996). Optokinetic stimulation influences the disturbed percepcion of body orientation in spatial neglect. Journal of Neurology, Neurosurgery and Psychiatry, 60, 217-220.

Karnath, H. O., Christ, K., \& Hartje, W. (1993). Decrease of contralateral neglect by neck muscle vibration and spatial orientation of trunk midline. Brain, 116, 383-396.

Karnath, H. O., Schenkel, P., \& Fischer, B. (1991). Trunk orientation as the determining factor of the "contralateral" deficit in the neglect syndrome and as the physical anchor of the internal representation of body orientation in space. Brain, 114, 1997-2014. 
Kinsbourne, M. (1993). Orientational bias model of unilateral neglect: Evidence from attentional gradients within hemispace. In I. H. Robertson \& J. C. Marshall (Eds.), Unilateral neglect: Clinical and experimental studies (pp. 69-85). Hove, UK: Lawrence Erlbaum Associates Ltd.

Lawson, I. R. (1962). Visual-spatial neglect in lesions of the right cerebral hemisphere. Neurology, 12, 23-33.

McGlynn, S., \& Schacter, D. (1989). Unwareness of deficits in neurophychological syndromes. Journal of Clinical and Experimental Neuropsychology, 11, 143-205.

Mesulam, M. M. (1981). A cortical network for directed attention and unilateral neglect. Annuals of Neurology, 10, 309-325.

Mesulam, M. M. (1990). Large-scale neurocognitive networks and distributed processing for attention, language and memory. Annuals of Neurology, 28, 597-613.

Paolucci, S., Antonucci, G., Guariglia, C., Magnotti, L., Pizzamiglio, L., \& Zoccolotti, P. (1996). Facilitatory effect of neglect rehabilitation on the recovery of left hemiplegic stroke patients: A cross-over study. Journal of Neurology, 243, 308-314.

Pardo, J. V., Fox, P. T., \& Raichle, M. E. (1991). Localization of a human system for sustained attention by positron emission tomography. Nature, 349, 61-64.

Pederson, P. M., Jogensen, H. S., Nakayama, H., Raaschou, H. O., \& Olsen, T. S. (1997). Hemineglect in acute stroke - incidence and prognostic implications: The Compenhagen stroke study. American Journal of Physical Medicine Rehabilitation, 76, 122-127.

Pizzamiglio, L., Antonucci, G., Judica, A., Montener, P., Razzano, C., \& Zoccolotti, P. (1992). Cognitive rehabbilitation of the hemineglect disorder in chronic patients with unilateral right brain damage. Journal of Clinical and Experimental Neuropsychology, 14 (6), 901-923.

Pizzamiglio, L., Frasca, R., Guariglia, C., Incoccia, C., \& Antonucci, G. (1990). Effect of optokinetic stimulation in patients with visual neglect. Cortex, 26, 535-540.

Posner, M. I. (1993). Interaction of arousal and selection in the posterior attention network. In A. Baddeley \& L. Weiskrantz (Eds.), Attention: selection, awareness and control (pp. 390-405). Oxford: Claredon Press.

Riddoch, M., \& Humphreys, G. (1983). The effect of cueing on unilateral neglect. Neuropsychologia, 21 (6), 589-599.

Robertson, I. H., Gray, J., Pentland, B., \& Waite, L. (1990). Microcomputer-based rehabilitation for unilateral left visual neglect: A randomised controlled trial. Archives of Physical Medicine and Rehabilitation, 71, 663-668.

Robertson, I. H., \& North, N. (1992). Spatio-motor cueing in unilateral neglect: The role of hemispace, hand and motor activation. Neuropsychologia, 30, 553-563.

Robertson, I. H., Tegnér, R., Tham, K., Lo, A., \& Nimmo-Smith, I. (1995). Sustained attention training for unilateral neglect: Theoretical and rehabilitation implications. Journal of Clinical and Experimental Neuropsychology, 17 (3), 416-430.

Rode, G., \& Perenin, M. (1994). Temporary remission of representation hemineglect through vestibular stimulation. NeuroReport, 5, 869-872.

Rubens, A. B. (1985). Caloric stimulation and unilateral visual neglect. Neurology, 35, 1019-1024. 
Soderback, I., Bengtsson, I., Ginsburg, E., \& Ekholm, J. (1992). Video feedback in occupational therapy: Its effect in patients with neglect syndrome. Archives of Physical Medicine \& Rehabilitation, 73, 1140-1146.

Stone, S. P., Halligan, P. W., Marshall, J. C., \& Greenwood, R. J. (1998). Unilateral neglect: A common but heterogeneous syndrome. Neurology, 50, 1902-1905.

Stone, S. P., Patel, P., Greenwood, R. J., \& Halligan, P. W. (1992). Measuring visual neglect in acute stroke and predicting its recovery: The visual neglect recovery index. Journal of Neurology, Neurosurgery and Psychiatry, 55, 431-436.

Sunderland, A., Wade, D. T., \& Hewer, R. L. (1987). The natural history of visual neglect after stroke. International Disabilities Studies, 9, 55-59.

Tham, K., \& Tegnér, R. (1997). Video feedback in the rehabilitation of patients with unilateral neglect. Archives of Physical Medicine \& Rehabilitation, 78, 410-413.

Vallar, G., Rusconi, M. L., Barozzi, S., Bernardini, B., Ovadia, D., Papagno, C., \& Cesarani, A. (1995). Improvement of left visuospatial hemineglect by left-sided transcutaneous electrical stimulation. Journal of the International Neuropsychological Society, 2, 452-459.

Vallar, G., Rusconi, M. L., \& Bernardini, B. (1996). Modulation of neglect hemianaesthesia by transcutaneous electrical stimulation. Journal of the International Neuropsychological Society, 2, 452-459.

Wagenaar, R., Van Wieringen, P., Netelenbos, J., Meijer, O., \& Kuik, D. (1992). The transfer of scanning training effects in visual attention after stroke: Five single case studies. Disability and Rehabilitation, 14, 51-60.

Webster, J., Jones, S., Blanton, P., Gross, R., Beissel, G., \& Wofford, J. (1984). Visual scanning training with stroke patients. Behaviour Therapy, 15, 129-143.

Weinberg, J., Diller, L., Gordon, W., Gerstman, L., Lieberman, A., Lakin, P., Hodges, G., \& Ezrachi, O. (1977). Visual scanning training effect on reading-related tasksin acquired right brain damage. Archives of Physical Medicine and Rehabilitation, 58, 479-486.

Wiart, L., Saintcome, A. B., Debelleix, X., Petit, H., Joseph, P. A., Mazaux, J. M., \& Barat, M. (1997). Unilateral neglect syndrome rehabilitation by trunk rotation and scanning training. Archives of Physical Medicine \& Rehabilitation, 78 (4), 424-429.

Unilateral spatial neglect: technics of rehabilitation (abstract) Neglect is the major cognitive deficit that results from a lesion on the right cerebral hemisphere. The clinical manifestations are very heterogeneous and bring to the patient serious repercussions on his quality of life. The fact that the more evident manifestations recover spontaneously during the first 10 days after lesion, could explain the generalized idea, among health staff, that neglect has always a favourable prognostic. In reality, the manifestations of neglect can persist, although subtly, for a long period. That's why the implementation of programs of rehabilitation is so important. The first techniques of rehabilitation for neglect were developed, intuitively, from the clinical practice. Later, a training of the visuospatial scanning was developed on a systematic way. Although this type of intervention may not permit a generalization for functional activities, is useful for training specific activities. A number of other techniques have been implemented to reduce the manifestations of neglect; however, the results are not conclusive. 